\title{
Arterial Levels of Oxidized Glutathione (GSSG) Reflect Oxidant Stress in Vivo ${ }^{1}$
}

\author{
Eddie K. ABdalla, B.S., Michael G. Caty, M.D., Karen S. Guice, M.D.,* \\ Daniel B. Hinshaw, M.D., ${ }^{*}$ AND KeITH T. Oldham, M.D. ${ }^{2}$ \\ Sections of Pediatric Surgery and General Surgery and ${ }^{*}$ Surgical Service Ann Arbor VAMC, Department of Surgery, \\ University of Michigan Medical School, Ann Arbor, Michigan 48109
}

Presented at the Annual Meeting of the Association for Academic Surgery, Louisville, Kentucky, November 15-18, 1989

Neutrophil-related, oxidant-mediated injury to the pulmonary microvasculature appears to follow endotoxemia, cutaneous thermal injury, and ischemia-reperfusion injury to the liver or intestine. Glutathione is an important endogenous intracellular oxygen radical scavenger. Plasma concentrations of oxidized glutathione (GSSG) reflect oxidant injury resulting from an overdose of certain oxidatively metabolized drugs. The purpose of this investigation was to evaluate plasma GSSG as an indicator of oxidant stress resulting from activation of the endogenous inflammatory response. An established model of neutrophil- and oxidant-related acute lung injury following intestinal ischemia and reperfusion in rats was used. Intestinal ischemia was induced by clip occlusion of the superior mesenteric artery (SMA) for $120 \mathrm{~min}$. Reperfusion resulted from SMA clip removal. Following reperfusion for 0,15 , or $120 \mathrm{~min}$, plasma GSSG levels in portal vein, inferior vena cava (IVC), and aorta were obtained. Plasma GSSG was undetectable in sham animals and those with intestinal ischemia alone. Following reperfusion, all plasma samples had significant elevations in GSSG. Aortic plasma GSSG after $15 \mathrm{~min}$ of reperfusion was significantly elevated compared to both portal vein and IVC plasma GSSG. These data suggest that oxidant stress after intestinal reperfusion is reflected by elevations in plasma GSSG. The step up in plasma GSSG across the pulmonary vascular bed, a site of known oxidant injury, suggests that plasma GSSG muy be a useful marker of oxidant stress in vivo, particularly with regard to the pulmonary microvasculature. This simple in vivo approach to assessing oxidant stress related to inflammatory tissue injury may have the potential to be of significant use in the clinical setting. (c) 1990 Academic Press, Inc.

\section{INTRODUCTION}

Neutrophil-related, oxidant-mediated injury to the pulmonary microvasculature has been reported following

\footnotetext{
${ }^{1}$ This work was sponsored in part by NIH Grant HL38141 and a VA Merit Review grant.

${ }^{2}$ To whom correspondence and reprint requests should be addressed at Section of Pediatric Surgery, University of Michigan Medical School, F7516 Mott Children's Hospital, Box 0245, Ann Arbor, MI 48109-0245.
}

endotoxemia [1-3], cutaneous thermal injury [4], and ischemia-reperfusion injury to the liver [5] or intestine [6]. Existing methods of assessing oxygen radical activity in vivo are cumbersome, indirect, and have not been clinically applicable [7]. In particular, it has not been feasible to quantify oxidant stress clinically using a plasma marker. A variety of indirect markers of lipid peroxidation including fluorescent products, conjugated dienes, and malondialdehyde have been proposed with limited enthusiasm $[7,8]$. However, some information has been accumulated in the toxicology literature suggesting that pharmacologically induced oxidant-mediated tissue injury (using agents such as carbon tetrachloride, acetaminophen, or paraquat) may be assessed more directly by using plasma glutathione determinations [9-13].

Oxygen radical formation in excess of normal physiologic limits appears to be one fundamental mechanism by which tissues are injured. Although highly reactive oxygen radicals result from normal metabolism, efficient endogenous enzymatic systems of removal normally prevent injury. In excess, however, these oxygen products may destroy lipid cell membranes, cause DNA strand breakage, and inhibit important cellular enzymes [14-16]. A critical intracellular component of this endogenous oxygen radical scavenging system is the tripeptide glutathione (GSH). Normally present in its reduced form, intracellular GSH is oxidized in the presence of oxygen radical species and is translocated into the blood in its oxidized form (GSSG) $[17,18]$. Arteriovenous differences in plasma GSSG levels across the liver after paraquat administration have been used to evaluate oxidant injury [9]. These data suggest that tissue injured by oxidants may be a source of plasma GSSG. Thus, plasma levels of oxidized glutathione may reflect oxygen radical activity in vivo. Systemic plasma GSSG levels might provide an index of whole-body oxidant stress. Arteriovenous differences in GSSG levels across a specific organ might reflect the contribution of that specific organ to plasma GSSG levels [19].

Given the large body of recent evidence for the importance of oxidant-mediated tissue injury resulting from activation of the endogenous inflammatory response, this study was designed to evaluate plasma GSSG as a marker 
of in vivo oxidant stress. The model used was one of adult respiratory distress syndrome (ARDS)-like acute lung injury induced by an ischemia-reperfusion injury to the rat intestine [6]. The data show that tissue injury resulting from activation of the endogenous inflammatory system produces measurable changes in plasma GSSG levels and that selective sampling may allow assessment of specific target organs.

\section{METHODS}

\section{Animal Model}

Male pathogen-free, Sprague-Dawley rats (150-250 g) (Charles River, Portage, MI) were used for all experiments. Experimental protocols were approved by the University of Michigan Committee on Use and Care of Animals. Anesthesia was administered by intramuscular (im) injection of $100 \mathrm{mg} / \mathrm{kg}$ ketamine hydrochloride. Midline laparotomy was performed and intestinal ischemia achieved by occlusion of the superior mesenteric artery (SMA) using a noncrushing Heifetz microvascular clip. Reperfusion was achieved by removal of the Heifetz clip at a second laparotomy following a second anesthetic ( $50 \mathrm{mg} / \mathrm{ml} \mathrm{ketamine} \mathrm{hydrochloride).} \mathrm{The} \mathrm{period} \mathrm{of} \mathrm{isch-}$ emia was $120 \mathrm{~min}$, followed by $0,15,60$, or $120 \mathrm{~min}$ of reperfusion. The model is characterized by hemodynamic stability and intestinal reperfusion has been confirmed by blood flow assessment [6]. Following reperfusion, blood samples were obtained from the portal vein, inferior vena cava (IVC), and descending thoracic aorta for analysis as outlined below. Sacrifice was by exsanguination at the designated time points. The heart and lungs were removed en bloc. While a spontaneous heart beat persisted, $20 \mathrm{ml}$ of sterile physiologic saline were infused into the right ventricle to wash residual blood from the pulmonary vascular bed. Both lungs were removed from the heart and hilar structures and frozen immediately in liquid nitrogen. Sham-operated control animals underwent identical preparation except that the SMA clip was not applied; therefore, no ischemia resulted.

\section{Glutathione Assay (Plasma)}

The method of Adams et al. [19] was modified as outlined. Whole blood was collected in heparin-coated syringes. For total GSH, $200 \mu \mathrm{l}$ of fresh whole blood was added to $200 \mu \mathrm{l}$ of a buffer containing $10 \mathrm{mM} 5,5^{\prime}$-dithiobis(2-nitrobenzoic acid) (DTNB), $17.5 \mathrm{mM}$ disodium EDTA, and $100 \mathrm{~m} M$ potassium phosphate, $\mathrm{pH}$ 7.5. Samples were mixed by tilting and then centrifuged $4 \mathrm{~min}$ at $2000 \mathrm{~g}$. Two hundred fifty microliters of the supernatant was stored on ice for analysis within $3-4 \mathrm{hr}$ or frozen at $-70^{\circ} \mathrm{C}$ for analysis within $48 \mathrm{hr}$. Freezing does not alter the assay (unpublished data).

To assay for GSSG, $200 \mu \mathrm{l}$ fresh whole blood was added to a buffer containing $10 \mathrm{mM} \mathrm{N}$-ethylmaleimide (NEM), $17.5 \mathrm{~m} M$ disodium EDTA, and $100 \mathrm{mM} \mathrm{K} \mathrm{K}_{2} \mathrm{H} \mathrm{PO}_{4}, \mathrm{pH}$ 6.5. Mixing and centrifugation were as above. NEM de- rivatizes all reduced GHS, leaving only GSSG for measurement by the enzymatic assay. The sample was then passed through a C18 Sep-pak cartridge (Waters Associates, Framingham, MA) which was prewashed with 1 $\mathrm{ml}$ methanol followed by $2 \mathrm{ml}$ distilled water. Each sample was rinsed from the cartridge with $1 \mathrm{ml}$ phosphate-imidazole buffer $\left(0.01 \mathrm{M} \mathrm{Na}_{2} \mathrm{HPO}_{4}\right.$ and $0.017 \mathrm{M}$ imidazole, $\mathrm{pH}$ 7.2). The eluate was placed on ice or frozen for analysis the next day.

A standard curve was generated using known amounts of GSH (Sigma, St. Louis, MO). Determination of GSH equivalents was made in plastic cuvettes containing 700 $\mu \mathrm{l} 0.3 \mathrm{~m} M$ DTNB and $700 \mu \mathrm{l} 0.4 \mathrm{~m} M$ NADPH (Sigma) containing 1.2 units/ml glutathione reductase (Sigma, Type III) to which $700 \mu$ l of the experimental sample was added for NEM-treated samples. For total GSH determination, $50-100 \mu \mathrm{l}$ of the plasma supernatant was used. Volume differences were corrected with the phosphateimidazole buffer. The difference in optical density was determined at $412 \mathrm{~nm}$ on an LKB Ultraspec II spectrophotometer (Biochrom, Model 4050, Cambridge, England). Concentrations of GSSG and total GSH were then determined by reverse linear regression against the standard curve.

\section{Glutathione Assay (Tissue)}

Frozen lung tissue was lyophilized for $24 \mathrm{hr}$ and stored at $-70^{\circ} \mathrm{C}$. At the time of assay, tissue was ground under liquid nitrogen with a mortar and pestle and weighed into 50-mg aliquots, mixed quickly with $1 \mathrm{ml}$ of a solution containing $2.5 \%$ sulfosalicylic acid in $0.2 \%$ Triton X-100, vortexed $15 \mathrm{sec}$, and centrifuged at $10,000 \mathrm{rpm}$ for $10 \mathrm{sec}$. The supernatant was removed and placed on ice. For the determination of tissue GSSG, the GSH in a sample was derivatized with $2 \mu \mathrm{l} 2$-vinylpyridine in $10 \mu \mathrm{l} 1 \mathrm{M}$ Tris at room temperature for $50 \mathrm{~min}$ and then frozen at $-70^{\circ} \mathrm{C}$ with the untreated supernatant. Assay of tissue total GSH and GSSG was thereafter identical to the plasma assay.

\section{Statistical Analysis}

Statistical evaluation throughout is by an analysis of variance using Tukey's multiple comparisons. Significance is defined as $P<0.05$ and individual comparisons are indicated in the text.

\section{RESUITS}

This model of intestinal ischemia and reperfusion yields a time-dependent, progressive intestinal injury that is associated with an acute pulmonary microvascular injury [6]. This lung injury is characterized by (1) significant reduction in lung tissue ATP levels following $15 \mathrm{~min}$ of reperfusion, (2) neutrophil sequestration in the pulmonary microvasculature as indicated by increasing myeloperoxidase concentrations in lung tissue and by quantitative morphometric evaluation, and (3) marked increases in lung microvascular permeability [6]. Both endotoxin and 
tumor necrosis factor appear to be involved in the pathogenesis of this injury [20]. The histologic characteristics of this lung injury are illustrated in Fig. 1. These include interstitial edema formation, endothelial cell blebbing with areas of focal necrosis, neutrophil infiltration, and intraalveolar fibrin deposition.

\section{Oxidized Glutathione Determinations in Plasma (Fig. 2) and Lung Tissue (Fig. 3)}

GSSG is not detectable in normal rat plasma (Fig. 2) or in normal human plasma (unpublished data). In addition, GSSG does not appear in plasma following intestinal ischemia alone. Following reperfusion of the gut, GSSG appears within $15 \mathrm{~min}$ in plasma. At this early time point, a significant ( $P=0.0339$ ) step up in plasma GSSG occurs between the portal venous blood (0.64 $\pm 0.20 \mu \mathrm{eq} /$ liter $)$ and the arterial blood $(2.04 \pm 0.62 \mu \mathrm{eq} /$ liter). Following $60 \mathrm{~min}$ of reperfusion, GSSG levels are further increased but the differential between venous and arterial plasma compartments no longer exists.
Lung tissue GSSG remained at low levels and was unchanged throughout the experimental periods (Fig. 3).

\section{Total Glutathione in Plasma (Fig. 4) and Lung Tissue (Fig. 5)}

Total GSH in plasma was statistically similar in the different vascular compartments in sham (0/0) animals and those subjected to ischemia alone (120/0) (Fig. 4). Following $15 \mathrm{~min}$ of intestinal reperfusion, elevations were seen in the aortic plasma $(66.4 \pm 14.7 \mu \mathrm{eq} /$ liter $)$ and portal venous plasma $(64.0 \pm 8.2 \mu \mathrm{eq} /$ liter $)$ that did not achieve statistical significance, compared to IVC plasma $(40.4$ $\pm 5.5 ; P>0.05)$. These sampling positions reflect effluent blood from the lungs and the intestine, respectively. The IVC, portal vein, and aorta GSH levels were also not significantly increased from those of sham $(0 / 0)$ or ischemic $(120 / 0)$ groups at this time. By $60 \mathrm{~min}$ of intestinal reperfusion, small elevations at all sites were present but a statistically significant increase was present only in the
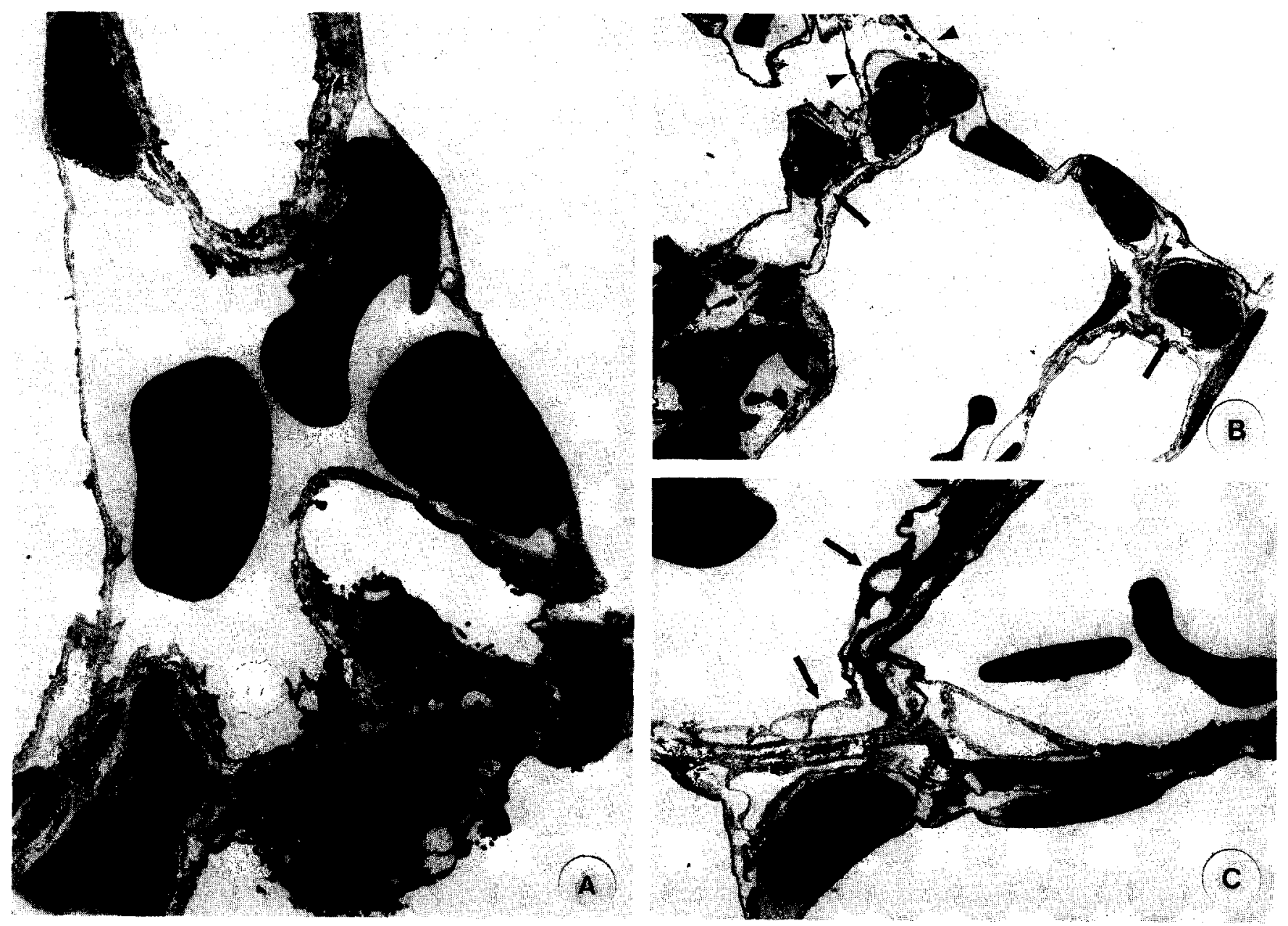

FIG. 1. The histological features of this acute lung injury are illustrated here. Normal (sham) lung histology is demonstrated in A (5600 $\times$ ). $B(2000 \times)$ and $C(4400 \times)$ are representative of lung histology after an intestinal injury of 120 min of ischemia and 120 min of reperfusion. Note the interstitial edema $(>$ ), endothelial cell blebbing $(\rightarrow)$, sequestration of neutrophils (PMN) in the microvasculature, and intraalveolar hemorrhage (rbc). 


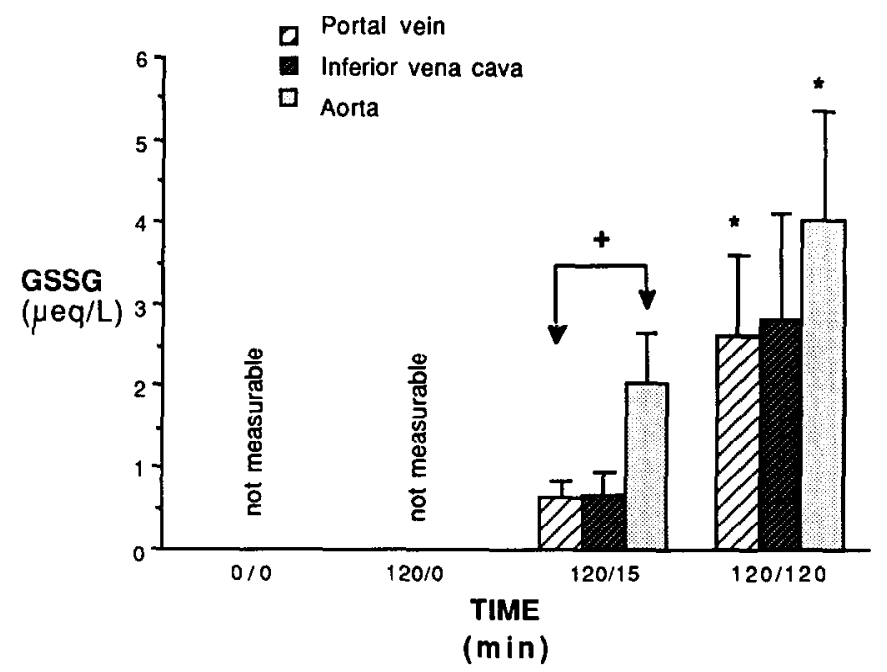

FIG. 2. Plasma GSSG levels from portal vein, inferior vena cava (IVC), and aorta are shown. Time points on the $x$-axis represent minutes of intestinal ischemia/minutes of intestinal reperfusion (e.g., 120/15 indicates $120 \mathrm{~min}$ of ischemia, $15 \mathrm{~min}$ of reperfusion). Following intestinal reperfusion, plasma GSSG is elevated. At 15 min of reperfusion a step up in GSSG occurs across the pulmonary vascular bed (between venous and arterial plasma). By $120 \mathrm{~min}$ of reperfusion, plasma GSSG is higher still, but differences among vascular compartments are no longer present. $\left({ }^{*} P=0.0009\right.$ compared to $0 / 0$ or $120 / 0,{ }^{+} P=0.0339$ as shown.)

IVC plasma. No intercompartmental differences were present.

Total GSH in lung tissue remained constant throughout the experimental periods (Fig. 5).

\section{DISCUSSION}

Normal cellular respiration involves the generation of toxic oxygen radicals. Ordinarily, they are quickly re-

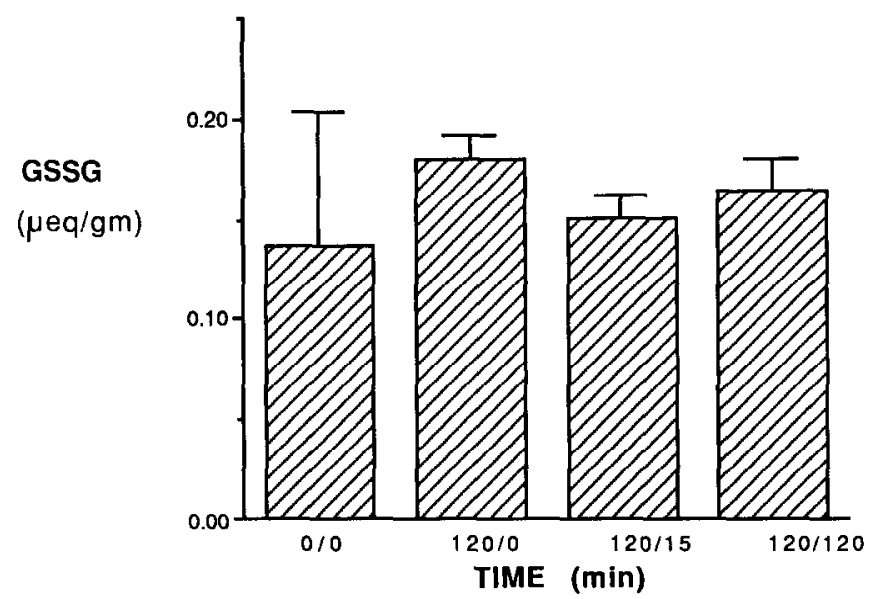

FIG. 3. Lung tissue GSSG remained at low levels and did not change during the experimental periods, suggesting rapid transfer of GSSG from the intracellular compartment to plasma after oxidation. Time points on the $x$-axis represent minutes of intestinal ischemia/minutes of intestinal reperfusion (e.g., 120/15 indicates $120 \mathrm{~min}$ of ischemia, $15 \mathrm{~min}$ of reperfusion). $(P>0.05$ for all time points.)

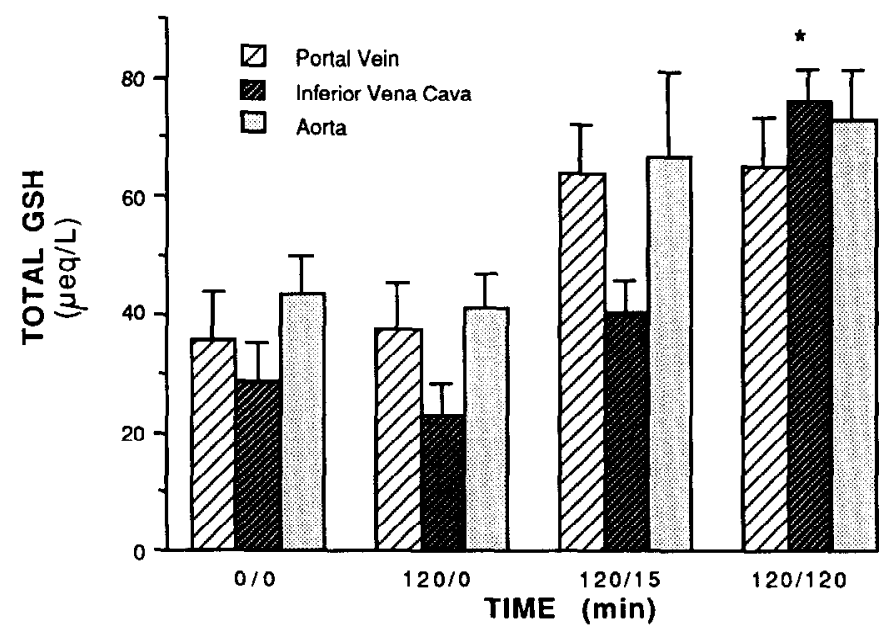

FIG. 4. Total plasma GSH in portal vein, inferior vena cava, and aorta is shown. Following intestinal reperfusion, elevations in total GSH occur that do not achieve statistical significance at the $120 / 15$ time point. These portal vein and aorta sampling sites reflect effluent blood from the intestine and lungs, respectively. By $60 \mathrm{~min}$ of reperfusion, total GSH in all compartments is slightly elevated but only the IVC elevation achieves statistical significance compared to the $0 / 0$ group. [Time points on the $x$-axis represent minutes of intestinal ischemia/ minutes of intestinal reperfusion (e.g., 120/15 indicates $120 \mathrm{~min}$ of ischemia, $15 \mathrm{~min}$ of reperfusion)]. ( ${ }^{*} P=0.0001$ compared to $0 / 0$ or $120 / 0$.)

moved from cells and from the circulation before they react with critical molecules such as the lipids in cell membranes and the proteins which regulate all cellular processes. Endogenous defenses against toxic oxygen radicals include a variety of scavenging enzyme systems. Glutathione is a ubiquitous, largely intracellular antioxidant that is believed to play a key role in the defense against oxygen-derived free radicals $[17,18]$. In the presence of oxygen radicals, reduced glutathione is oxidized to GSSG. In normal plasma, levels of GSSG are very low

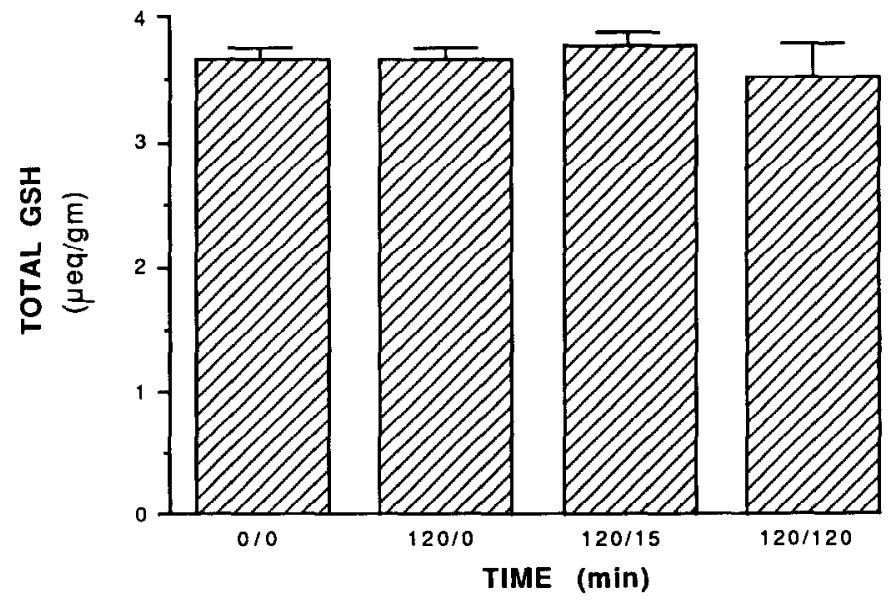

FIG. 5. Total GSH in lung tissue did not change during the experiental periods. 'Time points on the $x$-axis represent minutes of intestinal ischemia/minutes of intestinal reperfusion (e.g., 120/15 indicates $120 \mathrm{~min}$ of ischemia, $15 \mathrm{~min}$ of reperfusion). ( $P>0.05$ for all time points.) 
or undetectable by enzymatic assay [21]. It has been suggested that cells transport GSSG into the extracellular compartment to keep the intracellular concentrations of GSH and GSSG roughly constant [10, 22, 23]. Thus, plasma GSSG levels have theoretical appeal as a potential indicator of intracellular oxidant stress. Indeed, the liver has been shown to transport GSSG into blood following free radical generation induced by drug overdose [21]. A variety of drugs which are oxidatively metabolized are known to increase levels of GSSG in plasma $[10,11,12$, 13]. Finally, arteriovenous differences in GSSG after paraquat administration have been reported [9]. Collectively, these observations provide reason to expect that tissue injured oxidatively as a result of inflammation may be a source of plasma GSSG.

The lung injury generated by this animal model may be mediated in part by neutrophils. The evidence for this includes morphometric evaluation, myeloperoxidase accumulation in lung parenchyma, and increased pulmonary microvascular leak as previously reported [6]. Others have provided evidence that this type of pulmonary endothelial cell injury is neutrophil dependent and oxygen radical generated in vivo [24-27] and in vitro [28].

The data provided in Fig. 2 for GSSG in plasma represent a relatively direct approach for evaluation of oxidant stress in vivo. Significant increases in plasma GSSG occur with intestinal reperfusion. Given the body of literature which suggests that oxygen radicals participate in reperfusion injury to the intestine, this is not a surprising observation $[29,30]$. The significant step up in aortic GSSG suggests that an additional oxidant-injured "target" is interposed between venous and arterial compartments. The pulmonary microvasculature is a possible target capillary bed. This is consistent with previous data which suggest a role for sequestered neutrophils in alveolar capillaries [6] and with the work of others suggesting this common mechanism in other similar injuries $[1,24$, $25,26,31]$. The time-dependent rise in GSSG through 120 min of reperfusion suggests ongoing reperfusion injury. The loss of compartmental differences may reflect the fact that the circulation has had sufficient time to equilibrate and that the compartmentalization is, of course, incomplete. This suggests that discrete, organspecific, oxidant-mediated injuries may be localized by carly compartmental plasma sampling and that wholebody or multiple-organ oxidant stress may be assessed at later times. The data in Fig. 3 show that lung tissue GSSG is unaffected during oxidant stress and remains at low levels. This is consistent with the hypothesis that the generation of intracellular GSSG is rapidly followed by translocation into the plasma to preserve the intracellular environment.

Total plasma GSH, unlike GSSG, does not change significantly following reperfusion. Lung tissue total GSH and GSSG remain constant throughout the experimental period. The intracellular environment (GSH/GSSG ratio) is preserved. These data are in contrast to earlier work demonstrating a drop in total tissue GSH in a model of acute inflammatory injury to the lung [32]. Two factors may account for the different results. The earlier work was in rabbits, as opposed to rats in this study, and pulmonary injury was induced directly in the rabbit model whereas this model involved an indirect means of initiating the pulmonary injury. Thus, the plasma determinations of GSSG may represent a sensitive means of measuring what may be a more subtle oxidant stress. Whereas GSSG determinations are a measure of only oxidized glutathione, total GSH is a measure of the sum of oxidized plus reduced glutathione. Total glutathione levels may then reflect increases in GSSG, increased excretion of GSH out of cells into plasma, and perhaps altered capacity of the kidney to excrete GSH normally [10]. In light of these findings, plasma GSSG may be a more sensitive indicator of oxidant injury than total plasma glutathione or even tissue glutathione.

In summary, plasma GSSG appears to be a sensitive early indicator of oxidant injury. In contrast to total GSH levels, normal plasma GSSG levels are very low or undetectable, and any elevation of GSSG in plasma suggests pathology. These data suggest that GSSG determinations in plasma may reflect oxidant stress in vivo. Early sampling from specific vascular compartments may allow assessment of specific target organs; in particular, arteriovenous differences may reflect pulmonary microvascular injury. Later sampling particularly from systemic venous sites may reflect whole-body oxidant stress. The potential clinical use of this approach has great appeal. Further experimental and clinical studies are needed to confirm this preliminary investigation.

\section{ACKNOWLEDGMENT}

The authors thank Jim Wan, Ph.D., of the University of Michigan Department of Biostatistics for his assistance with data analysis.

\section{REFERENCES}

1. Demling, R., Lalonde, C., Seekamp, A., and Fiore, N. Endotoxin causes hydrogen peroxide-induced lung lipid peroxidation and prostanoid production. Arch. Surg. 123: 1337, 1988.

2. Bisio, J. M., Breen, R. E., Connell, R. S., and Harrison, M. W. Pulmonary capillary endothelial dysfunction in hypoxia and endotoxemia: A biochemical and electron microscopic study. $J$. Trauma 23(8): 730, 1983.

3. Simons, R. K., Maier, R. V., and Lennard, E. S. Neutrophil function in a rat model of endotoxin-induced lung injury. Arch. Surg. 122: $197,1987$.

4. Till, G. O., Beauchamp, C., Menapace, D., Tourtellotte, W., Kunkel, R., Johnson, K. J., and Ward, P. A. Oxygen radical dependent lung danage following thermal injury of rat skin. $J$. Trauma 23: 269, 1983.

5. Colletti, L. M., Burtch, G. D., Remick, D. G., Kunkel, S. L., Strieter, R. M., Guice, K. S., Oldham, K. T., and Campbell, D. A. Production of tumor necrosis factor alpha and the development of a pulmonary capillary injury following hepatic ischemia-reperfusion. Transplantation, in press.

6. Schmeling, D. J., Caty, M. G., Oldham, K. T., Guice, K. S., and Hinshaw, D. B. Evidence for a neutrophil-related acute long injury following intestinal ischemia-reperfusion injury. Surgery 106(2): $195,1989$. 
7. Taylor, A. E., and Townsley, M. I. Assessment of oxygen radical tissue damage. In A. E. Taylor, S. Matalon, and P. Ward (Eds.), Physiology of Oxygen Radicals. Williams \& Wilkins, Baltimore, 1986.

8. Ward, P. A., Till, G. O., Hatherill, J. R., Annesley, T. M., and Kunkel, R. G. Systemic complement activation, lung injury, and products of lipid peroxidation. J. Clin. Invest. 76: 517, 1985.

9. Adams, J. R., Jr., Lauterburg, B. H., and Mitchell, J. R. Plasma glutathione disulfide as an index of oxidant stress in vivo: Effects of $\mathrm{CCl}_{4}$, dimethylnitrosamine, nitrofurantoin, metronidazole, doxorubicin and diquat. Res. Commun. Chem. Pathol. Pharmacol. 46(3): 401, 1984.

10. Griffith, O. W., and Meister, A. Glutathione: Interorgan translocation, turnover, and metabolism. Proc. Natl. Acad. Sci. USA 76(11): 5606, 1979.

11. Lauterburg, B. H., Corcoran, G. B., and Mitchell, J. R. Mechanism of action of $N$-acetylcysteine in protection against the hepatotoxicity to acetaminophen in rats in vivo. J. Clin. Invest. 71: 980, 1983.

12. Reed, D. J. Regulation of reductive processes by glutathione. Biochem. Pharmacol. 35(1): 7, 1986.

13. Vina, J., Romero, F. J., Estrela, J. M., and Vina, J. R. Effect of acetaminophen (paracetamol) and its antagonist on glutathione (GSH) content in rat liver. Biochem. Pharmacol. 29: 1968, 1969.

14. Taylor, A. E., Matalon, S., and Ward, P. Physiology of Oxygen Radicals. Williams \& Wilkins, Baltimore, 1986.

15. Hyslop, P. A., Hinshaw, D. B., Halsey, W. A., Schraufstatter, I. U., Sauerheber, R. D., Spragg, R. G., Jackson, J. H., and Cochrane, C. G. Mechanisms of oxidant-mediated cell injury: The glycolytic and mitochondrial pathways of ADP phosphorylation are major intracellular targets inactivated by hydrogen peroxide. J. Biol. Chem. 263: 1665, 1988.

16. Schraufstatter, I. U., Hinshaw, D. B., Hyslop, P. A., Spragg, R. G., and Cochrane, C. G. Oxidant injury of cells: DNA strand breaks activate poly-ADP-ribose polymerase and lead to depletion of nicotanamide adenine dinucleotide. J. Clin. Invest. 77: 1312, 1986.

17. Meister, A. Selective modification of glutathione metabolism. Science 220: 472, 1983.

18. Meister, A. Glutathione. The Liver: Biology and Pathology, 2nd ed. In I. M. Arias, W. B. Jakoby, H. Popper, D. Schachter, and D. A. Shafritz, (Eds.), Raven Press, New York, 1988.

19. Adams, J. D., Jr., Bernhard, H., Lauterburg, B. H., and Mitchell, J. R. Plasma glutathione and glutathione disulfide in the rat: Reg- ulation and response to oxidative stress. J. Pharmacol. Exp. Ther. 227(3): 749, 1983.

20. Caty, M. G., Schemling, D. J., Guice, K. S., Oldham, K. T., Remick, D. G., and Kunkel, S. L. Evidence for TNF-related pulmonary microvascular injury following intestinal ischemia-reperfusion. Ann. Surg., in press.

21. Lauterburg, B. H., Smith, C. V., Hughes, H., and Mitchell, J. R. Biliary excretion of glutathione and GSSG in rat: Regulation and response to oxidative stress. J. Clin. Invest. 73: 124, 1984.

22. Arias, I. M., and Jaoby, W. B. (Eds.). GSH: Metabolism and Function. Kork Foundation Series, Vol. 6, p. 1. Rave, NY, 1976.

23. Gregus, Z., Stein, A. F., and Klaassen, C. D. Age dependent biliary excretion of glutathione-related thiols in rats: Role of $\gamma$-glutamyl transferase. Gastrointest. Liver Physiol. 16: G86, 1987.

24. Klausner, J. M., Anner, H., Paterson, I. S., Kobzik, L., Valeri, C. R., Shepro, D., and Hechtman, H. B. Lower torso ischemiainduced lung injury is leukocyte dependent. Ann. Surg. 208(6): $761,1988$.

25. Till, G. O., Johnson, K. J., Kunkel, R., and Ward, P. A. Intravascular activation of complement and acute lung injury: Dependency on neutrophils and toxic oxygen metabolites. J. Clin. Invest. 69: $1126,1982$.

26. Till, G. O., and Ward, P. A. Oxygen radicals in complement and neutrophil-mediated acute lung injury. J. Free Radicals Biol. Med. 1: $163,1985$.

27. Ward, P. A., Johnson, K. J., and Till, G. O. Oxygen radicals and mirrovascular injury of lungs and kidney. Acta Physiol. Scand. 548: 1986 .

28. Varani, J., Fliegiel, S. E. G., Till, G. O., Kunkel, R. G., Ryan, U. S., and Ward, P. A. Pulmonary endothelial cell killing by human neutrophils: Possible involvement of hydroxyl radical. Lab. Invest. 53: 656,1985 ,

29. Granger, D. N., Rutili, G., and McCord, J. M. Superoxide radical in feline intestinal ischemia. Gastroenterology 81: 22, 1981.

30. Granger, D. N., Hollworth, M. E., and Parks, D. A. Ischemiareperfusion injury: Role of oxygen-derived free radicals. Acta Physiol. Scand. (Suppl.) 548: 47, 1986.

31. Guice, K. S, Oldham, K. T., Caty, M. G., JOhnson, K. J., and Ward, P. A. Neutrophil dependent oxygen radical mediated lung injury associated with acute pancreatitis. Ann. Surg. 210: 740, 1989.

32. Schraufstatter, I. U., Revak, S. D., and Cochrane, C. G. Proteases and oxidants in experimental pulmonary inflammatory injury. $J$. Clin. Invest. 73: 1175, 1984. 